\title{
SOLUTIONS FOR THE $P(X)$-LAPLACIAN WITH DEPENDENCE ON THE GRADIENT
}

\author{
R. Ayazoglu (Mashiyev), S. Akbulutb, E. Akkoyunluc
}

ABSTRACT. This paper is concerned with the existence of nontrivial solutions for $p(x)$ Laplacian equations with gradient dependence

$$
\left\{\begin{array}{l}
-\operatorname{div}\left(|\nabla u|^{p(x)-2} \nabla u\right)+|u|^{p(x)-2} u=f\left(x, u,|\nabla u|^{p(x)-2} \nabla u\right) \text { in } \Omega, \\
u=0 \text { on } \partial \Omega
\end{array}\right.
$$

The techniques are based on an iterative scheme of Mountain Pass "approximated" solutions.

2010 Mathematics Subject Classification: 35J35; 35J60; 35J70; 35D30.

Keywords: Variable exponent Lebesgue-Sobolev spaces; $p(x)$-Laplacian; Iteration methods; Mountain Pass theorem

\section{INTRODUCTION}

In the present paper we study the existence of solutions of the problem

$$
\left\{\begin{aligned}
& -\operatorname{div}\left(|\nabla u|^{p(x)-2} \nabla u\right)+|u|^{p(x)-2} u=f\left(x, u,|\nabla u|^{p(x)-2} \nabla u\right) \text { in } \Omega, \\
u & =0 \text { on } \partial \Omega
\end{aligned}\right.
$$

where $\Omega \subset \mathbb{R}^{N}$ is a bounded smooth domain, $1<p(x)<2$ for any $x \in \bar{\Omega}$ and $f$ is a continuous function which obeys some specific conditions. Since the nonlinearity $f$ depends on the gradient of the solution, equation $(\mathbf{P})$ is not variational. Therefore, the well developed critical point theory cannot be applied directly. For this reason, there have been several works interested with the semilinear problem

$$
\left\{\begin{array}{l}
-\triangle u=f(u, \nabla u) \text { in } \Omega \\
u=0 \text { on } \partial \Omega
\end{array}\right.
$$

in a bounded domain $\Omega$ of $\mathbb{R}^{N}$, using method of sub and supersolutions, topological degree and priory bounds on the possible solutions; see, for instance, [20,23]. The case involving 
the $p$-Laplacian operator $\Delta_{p} u:=\operatorname{div}\left(|\nabla u|^{p-2} \nabla u\right)$, where $p>1$ is a real constant, was studied in $[15,18,21]$, in which the authors also used method of sub and supersolutions, topological degree and blow-up arguments. Recently, some new and interesting methods have been developed by different authors for problem (1.1). In [12], D.G. de Figueiredo et al. developed a quite different method of variational type for the semilinear elliptic problem

$$
\left\{\begin{array}{l}
-\triangle u=f(x, u, \nabla u) \text { in } \Omega, \\
u=0 \text { on } \partial \Omega
\end{array}\right.
$$

where $\Omega \subset \mathbb{R}^{N}(N \geq 3)$ is a bounded smooth domain. In this paper, the used technique consisted of associating (1.2) a family of semilinear elliptic problems with no dependence on the gradient of the solutions, which is variational, and iterative scheme. Under the assumptions that $f$ has a superlinear subcritical growth at zero and at infinity with respect to the second variable, they obtained the existence of a positive and a negative solutions of (1.2) by using the Mountain Pass theorem and iterative technique. Later, in [13] G. M. Figueiredo applied this method to a quasilinear elliptic problem

$$
-\triangle_{p} u+|u|^{p-2} u=f\left(u,|\nabla u|^{p-2} \nabla u\right) \text { in } \mathbb{R}^{N},
$$

where $1<p<N$ and the nonlinearity $f: \mathbb{R} \times \mathbb{R}^{N} \rightarrow \mathbb{R}$ is a continuous function depending on the gradient of the solution, and obtained a positive solution for (1.3).

Motivated from the above mentioned papers, especially $[12,13]$, we consider problem $(\mathbf{P})$. Further, as far as we know, there is only one paper which deals with an elliptic equation with variable exponent with dependence on the gradient of the solutions [25], and the present paper is the second.

Problem $(\mathbf{P})$ involves the term $\triangle_{p(x)} u:=\operatorname{div}\left(|\nabla u|^{p(x)-2} \nabla u\right)$ which is known as $p(x)$-Laplacian operator. The $p(x)$-Laplacian operator is a natural generalization of the $p$-Laplacian operator. The main difference between them is that $p$-Laplacian operator is $(p-1)$-homogenous, but the $p(x)$-Laplacian operator, when $p(x)$ is not constant, is not homogeneous. This causes many problems, some classical theories and methods, such as the theory of Sobolev spaces, are not applicable. Moreover, the nonlinear problems involving the $p(x)$-Laplacian operator are extremely attractive because they can be used to model dynamical phenomena which arise from the study of electrorheological fluids or elastic mechanics. Problems with variable exponent growth conditions also appear in the modelling of stationary thermo-rheological viscous flows of non-Newtonian fluids and in the mathematical description of the processes filtration of an ideal barotropic gas through a porous medium. The detailed application backgrounds of the $p(x)$-Laplacian can be found in $[3,6,22,25]$ and references therein.

Noted that in problem $(\mathbf{P})$ if $p(x) \in(1,2)$, this equation describes processes of fast diffusion, the case $p(x)>2$ corresponds to slow diffusion and the case $p(x)=2$ linear diffusion. In this paper, we will discuss the case of $p(x) \in(1,2), x \in \bar{\Omega}$. 


\section{Preliminaries}

We state some basic properties of the variable exponent Lebesgue and Sobolev spaces $L^{p(x)}(\Omega)$ and $W^{1, p(x)}(\Omega)$, where $\Omega \subset \mathbb{R}^{N}$ is a bounded domain (for details, see, e.g., $[7,8,9,16])$.

Set $C_{+}(\bar{\Omega})=\{h: h \in C(\bar{\Omega}), h(x)>1\}$ for all $x \in \bar{\Omega}$.

Define $h^{-}=\min _{x \in \bar{\Omega}} h(x)$ and $h^{+}=\max _{x \in \bar{\Omega}} h(x), \forall h \in C_{+}(\bar{\Omega})$. For any $p \in C_{+}(\bar{\Omega})$, we define the variable exponent Lebesgue space by

$$
L^{p(x)}(\Omega)=\left\{u \mid u: \Omega \rightarrow \mathbb{R} \text { is measurable, } \int_{\Omega}|u(x)|^{p(x)} d x<\infty\right\},
$$

then $L^{p(x)}(\Omega)$ endowed with the norm

$$
|u|_{p(x)}=\inf \left\{\lambda>0: \int_{\Omega}\left|\frac{u(x)}{\lambda}\right|^{p(x)} d x \leq 1\right\} .
$$

The modular of $L^{p(x)}(\Omega)$ which is the mapping $\rho_{p(x)}: L^{p(x)}(\Omega) \rightarrow \mathbb{R}$ is defined by

$$
\rho_{p(x)}(u)=\int_{\Omega}|u|^{p(x)} d x
$$

for all $u \in L^{p(x)}(\Omega)$.

Proposition 2.1. $[8,16]$ If $u, u_{n} \in L^{p(x)}(\Omega)(n=1,2, \ldots)$, then the following statements are equivalent:

(i) $\lim _{n \rightarrow \infty}\left|u_{n}-u\right|_{p(x)}=0$;

(ii) $\lim _{n \rightarrow \infty} \rho_{p(x)}\left(u_{n}-u\right)=0$;

(iii) $u_{n} \rightarrow u$ in measure in $\Omega$ and $\lim _{n \rightarrow \infty} \rho_{p(x)}\left(u_{n}\right)=\rho_{p(x)}(u)$.

Proposition 2.2. $[8,16]$ If $u, u_{n} \in L^{p(x)}(\Omega)(n=1,2, \ldots)$, we have

$(i)|u|_{p(x)}<1(=1 ;>1) \Leftrightarrow \rho_{p(x)}(u)<1(=1 ;>1)$;

$(i i)|u|_{p(x)}>1 \Longrightarrow|u|_{p(x)}^{p^{-}} \leq \rho_{p(x)}(u) \leq|u|_{p(x)}^{p^{+}} ;|u|_{p(x)}<1 \Longrightarrow|u|_{p(x)}^{p^{+}} \leq \rho_{p(x)}(u) \leq$ $|u|_{p(x)}^{p^{-}}$

(iii) $\lim _{n \rightarrow \infty}\left|u_{n}\right|_{p(x)}=0 \Leftrightarrow \lim _{n \rightarrow \infty} \rho_{p(x)}\left(u_{n}\right)=0 ; \lim _{n \rightarrow \infty}\left|u_{n}\right|_{p(x)}=\infty \Leftrightarrow \lim _{n \rightarrow \infty} \rho_{p(x)}\left(u_{n}\right)=\infty$.

The variable exponent Sobolev space $W^{1, p(x)}(\Omega)$ is defined by

$$
W^{1, p(x)}(\Omega)=\left\{u \in L^{p(x)}(\Omega):|\nabla u| \in L^{p(x)}(\Omega)\right\},
$$

with the norm

$$
\|u\|=|u|_{p(x)}+|\nabla u|_{p(x)},
$$


for all $u \in W^{1, p(x)}(\Omega)$.

Denote by $W_{0}^{1, p(x)}(\Omega)$ the closure of $C_{0}^{\infty}(\Omega)$ in $W^{1, p(x)}(\Omega)$; we know that $|\nabla u|_{p(x)}$ is an equivalent norm on $W_{0}^{1, p(x)}(\Omega)$. Moreover, it is well known that if $1<p^{-} \leq p^{+}<\infty$, then spaces $L^{p(x)}(\Omega), W^{1, p(x)}(\Omega)$ and $W_{0}^{1, p(x)}(\Omega)$ are separable and reflexive Banach spaces.

If we consider $\sigma_{p(x)}(u)=\int_{\Omega}\left(|\nabla u|^{p(x)}+|u|^{p(x)}\right) d x$ instead $\rho_{p(x)}(u)$, then the statements of Proposition 2.1 and Proposition 2.2 also hold for $u, u_{n} \in W^{1, p(x)}(\Omega)$.

Proposition 2.3. [7] Let $p(x)>1$ for all $x \in \Omega$ and $\frac{1}{p(x)}+\frac{1}{p^{\prime}(x)}=1$. Then, for all $a, b \geq 0$

$$
a b \leq \frac{a^{p(x)}}{p(x)}+\frac{b^{p^{\prime}(x)}}{p^{\prime}(x)} .
$$

Proposition 2.4. [7] If $p \in C_{+}(\bar{\Omega})$, the conjugate space of $L^{p(x)}(\Omega)$ is $L^{p^{\prime}(x)}(\Omega)$, where $\frac{1}{p^{\prime}(x)}+\frac{1}{p(x)}=1$. For any $u \in L^{p(x)}(\Omega)$ and $v \in L^{p^{\prime}(x)}(\Omega)$, we have

$$
\left|\int_{\Omega} u v d x\right| \leq\left(\frac{1}{p^{-}}+\frac{1}{\left(p^{-}\right)^{\prime}}\right)|u|_{p(x)}|v|_{p^{\prime}(x)} \leq 2|u|_{p(x)}|v|_{p^{\prime}(x)} .
$$

Proposition $2.5[8,16](i)$. Assume that the boundary $\partial \Omega$ of $\Omega$ possesses the cone property, and $p \in C(\bar{\Omega})$. If $q \in C(\bar{\Omega})$ and $1 \leq q(x)<p^{*}(x)$ for any $x \in \bar{\Omega}$, then $W^{1, p(x)}(\Omega) \hookrightarrow \hookrightarrow L^{q(x)}(\Omega)$.

(ii) If $p, q \in C(\bar{\Omega})$ and $p(x) \leq q(x)$ for any $x \in \bar{\Omega}$, then $W^{1, p(x)}(\Omega) \hookrightarrow L^{q(x)}(\Omega)$, and also there is a constant $c>0$ such that

$$
|u|_{q(x)} \leq c\|u\|, \forall u \in W_{0}^{1, p(x)}(\Omega) .
$$

Proposition 2.6. The operator $L$ satisfies the following propositions:

(i) $L: W_{0}^{1, p(x)}(\Omega) \rightarrow\left(W_{0}^{1, p(x)}(\Omega)\right)^{*}$ is a continuos, bounded and strictly monotone operator;

(ii) $L$ is a mapping of type $\left(S_{+}\right)$, i.e., if $u_{n} \rightarrow u$ (weakly) in $W_{0}^{1, p(x)}(\Omega)$, and

$$
\varlimsup_{n \rightarrow \infty}\left(L\left(u_{n}\right)-L(u), u_{n}-u\right) \leq 0,
$$

then $u_{n} \rightarrow u$ (strongly) in $W_{0}^{1, p(x)}(\Omega)$.

\section{MAin RESUltS}

First, we state the assumptions imposed on the nonlinearity $f$, which appears in problem $(\mathbf{P})$. Let $f: \bar{\Omega} \times \mathbb{R} \times \mathbb{R}^{N} \rightarrow \mathbb{R}$ is a continuous function which satisfies the following conditions: 
R. Ayazoglu (Mashiyev), S. Akbulutb, E. Akkoyunluc - Solutions for ...

$\left(\mathbf{f}_{1}\right)$

$$
f\left(x, t,|\xi|^{p(x)-2} \xi\right)=0 \forall t \leq 0, \forall(x, \xi) \in \bar{\Omega} \times \mathbb{R}^{N}
$$

$\left(\mathbf{f}_{2}\right)$

$$
\lim _{t \rightarrow 0} \frac{f\left(x, t,|\xi|^{p(x)-2} \xi\right)}{|t|^{p(x)-1}}=0 \text { uniformly for } x \in \bar{\Omega} \text { and } \xi \in \mathbb{R}^{N}
$$

$\left(\mathbf{f}_{3}\right)$

$$
\lim _{t \rightarrow \infty} \frac{f\left(x, t,|\xi|^{p(x)-2} \xi\right)}{|t|^{p(x)-1}}=0 \text { uniformly for } x \in \bar{\Omega} \text { and } \xi \in \mathbb{R}^{N},
$$

where $p(x)<p^{*}(x) \forall x \in \bar{\Omega}$, and $p^{*}(x)$ is the Sobolev critical exponent given by

$$
p^{*}(x)=\left\{\begin{array}{cc}
\frac{N p(x)}{N-p(x)} & \text { if } p(x)<N \\
+\infty & \text { if } p(x) \geq N
\end{array}\right.
$$

$\left(\mathbf{f}_{4}\right)$ (Ambrosetti-Rabinowitz's condition). There exists $\theta>p^{+}$and $t_{0}>0$ such that

$$
0<\theta F\left(x, t,|\xi|^{p(x)-2} \xi\right)=\int_{0}^{t} f\left(x, t,|\xi|^{p(x)-2} \xi\right) d t \leq t f\left(x, t,|\xi|^{p(x)-2} \xi\right),
$$

for all $|t| \geq t_{0}, x \in \bar{\Omega}$ and $\xi \in \mathbb{R}^{N}$.

$\left(\mathbf{f}_{5}\right)$ There exists constants $a_{1}, a_{2}>0$ such that

$$
F\left(x, t,|\xi|^{p(x)-2} \xi\right) \geq a_{1}|t|^{\theta}-a_{2}, \forall x \in \bar{\Omega}, \xi \in \mathbb{R}^{N}
$$

$\left(\mathbf{f}_{6}\right)$ There exists constants $L_{1}=L_{\rho_{1}}$ and $M>0$ such that

$$
\left|f\left(x, t_{1},|\xi|^{p(x)-2} \xi\right)-f\left(x, t_{2},|\xi|^{p(x)-2} \xi\right)\right| \leq L_{1}\left|t_{1}-t_{2}\right|^{p(x)-1}-\frac{M}{\left|t_{1}-t_{2}\right|}, 1<p(x)<2,
$$

for all $t_{1}, t_{2} \in\left[0, \rho_{1}\right]\left(t_{1} \neq t_{2}\right)$ and for all $|\xi| \leq \rho_{2}$.

$\left(\mathbf{f}_{7}\right)$ There exists constant $L_{2}=L_{\rho_{2}}$

$$
\left|f\left(x, t,\left|\xi_{1}\right|^{p(x)-2} \xi_{1}\right)-f\left(x, t,\left|\xi_{2}\right|^{p(x)-2} \xi_{2}\right)\right| \leq L_{2}\left|\xi_{1}-\xi_{2}\right|^{p(x)-1},
$$

for all $t \in\left[0, \rho_{1}\right]$ and for all $\left|\xi_{1}\right|,\left|\xi_{2}\right| \leq \rho_{2}$, where $\rho_{1}$ and $\rho_{2}$ depend on $p^{+}$and $\theta$ given in the previous assumptions.

Moreover, in the proof of the main result related to problem $(\mathbf{P})$, we use the well-known vector inequalities (see [17]) 
R. Ayazoglu (Mashiyev), S. Akbulutb, E. Akkoyunluc - Solutions for ...

$$
\left(|\eta|^{p(x)-2} \eta-|\psi|^{p(x)-2} \psi\right) \cdot(\eta-\psi) \geq\left(p^{-}-1\right) \frac{|\eta-\psi|^{2}}{(|\eta|+|\psi|)^{2-p(x)}}, 1<p(x)<2,
$$

for all $x \in \bar{\Omega}$ and $\eta, \psi \in \mathbb{R}^{N}$, where " ." is the inner product usual in $\mathbb{R}^{N}$.

The following theorem is crucial to get the regularity of the solutions obtained in the present paper.

Theorem A. (a) [10, Theorem 4.1] If $f$ satisfies the growth condition

$$
\left|f\left(x, t,|\xi|^{p(x)-2} \xi\right)\right| \leq C_{1}|t|^{p(x)-1}+C_{2}|t|^{q(x)-1}+C_{3}, \forall(x, t, \xi) \in \bar{\Omega} \times \mathbb{R} \times \mathbb{R}^{N},
$$

where $C_{1}, C_{2}, C_{3}>0$ and $q \in C_{+}(\bar{\Omega})$ such that $q(x)<p^{*}(x)$ for all $x \in \bar{\Omega}$, then $u \in L^{\infty}(\Omega)$ for every weak solution $u$ of $(\mathbf{P})$.

(b) $\left[10\right.$, Theorem 4.4] Let $u \in W_{0}^{1, p(x)}(\Omega) \cap L^{\infty}(\Omega)$ be a solution of $(\mathbf{P})$. If the function $p$ is log-Hölder continuous on $\bar{\Omega}$, i.e., there exists a positive constant $H$ such that

$$
|p(x)-p(y)| \leq \frac{H}{-\log |x-y|} \text { for } x, y \in \bar{\Omega} \text { with }|x-y| \leq \frac{1}{2},
$$

then $u \in C^{0, \alpha}(\bar{\Omega})$ for some $\alpha \in(0,1)$.

(c) $\left[11\right.$, Theorem 1.2] Let $u \in W_{0}^{1, p(x)}(\Omega) \cap L^{\infty}(\Omega)$ be a solution of $(\mathbf{P})$. If the function $p$ is Hölder continuous on $\bar{\Omega}$, i.e., there exists a positive constant $H$ such that

$$
|p(x)-p(y)| \leq H|x-y|^{\alpha} \text { for } x, y \in \bar{\Omega},
$$

then $u \in C^{1, \alpha}(\bar{\Omega})$ for some $\alpha \in(0,1)$.

Theorem 3.1. Assume the conditions $\left(\mathbf{f}_{1}\right)-\left(\mathbf{f}_{7}\right)$ hold. If in addition $p$ also satisfies (3.3) and $1<p(x)<2$ for all $x \in \bar{\Omega}$, then problem $(\mathbf{P})$ has a positive solution provided

$$
L_{1} L_{3} p^{-}+L_{2} L_{4} p^{+}<\frac{p^{-}\left(p^{-}-1\right)}{2},
$$

where $1<p^{-} \leq p^{+}<2$ and $L_{3}, L_{4} \geq 1$ are real numbers.

Moreover the solution obtained is of class $C^{1, \alpha}(\bar{\Omega})$ for some $\alpha \in(0,1)$.

A similar result was obtained at [2] in the case of $2 \leq p(x)<N$ for all $x \in \bar{\Omega}$.

The proof of Theorem 3.1 is broken into several parts listed as follows. Actually problem $(P)$ is not variational, due to the presence of the gradient in $\mathrm{f}$, but ifone "freezes" the gradient variable, that is one fixes any $w$ in the variable exponent Sobolev space $W_{0}^{1, p(x)}(\Omega)$ and considers the problem

$$
\left\{\begin{array}{c}
-\operatorname{div}\left(|\nabla u|^{p(x)-2} \nabla u\right)+|u|^{p(x)-2} u=f\left(x, u,|\nabla w|^{p(x)-2} \nabla w\right) \text { in } \Omega \\
u=0 \text { on } \partial \Omega
\end{array}\right.
$$


The idea is to consider a class of problems such as $\left(\mathbf{P}_{w}\right)$ through an iterative scheme where any "approximated" problem has a positive Mountain Pass solution, say $u_{n}$. Since problem $\left(\mathbf{P}_{w}\right)$ is in a variational setting, the weak solutions of it are the critical points of the corresponding functional $I_{w}: W_{0}^{1, p(x)}(\Omega) \rightarrow \mathbb{R}$ defined by

$$
I_{w}(u)=\int_{\Omega} \frac{|\nabla u|^{p(x)}+|u|^{p(x)}}{p(x)} d x-\int_{\Omega} F\left(x, u,|\nabla w|^{p(x)-2} \nabla w\right) d x .
$$

In a standard way we can prove that $I_{w} \in C^{1}\left(W_{0}^{1, p(x)}(\Omega), \mathbb{R}\right)$. Our first results will be about the solvability of $\left(\mathbf{P}_{w}\right)$ and bound estimates of its solutions.

We deduce that $I_{w} \in C^{0}\left(W_{0}^{1, p(x)}(\Omega), \mathbb{R}\right) \cap C^{1}\left(W_{0}^{1, p(x)}(\Omega) \backslash\{0\}, \mathbb{R}\right)$ with

$$
\left\langle I_{w}^{\prime}\left(u_{w}\right), \varphi\right\rangle=\int_{\Omega}|\nabla u|^{p(x)-2} \nabla u \nabla \varphi d x-\int_{\Omega} f\left(x, u,|\nabla w|^{p(x)-2} \nabla w\right) \varphi d x
$$

for all $u \in W_{0}^{1, p(x)}(\Omega) \backslash\{0\}, \varphi \in W_{0}^{1, p(x)}(\Omega)$.

We say that $u \in W_{0}^{1, p(x)}(\Omega)$ is a weak solution of $\left(\mathbf{P}_{w}\right)$ if

$$
\int_{\Omega}|\nabla u|^{p(x)-2} \nabla u \nabla \varphi d x+\int_{\Omega}|u|^{p(x)-2} u \varphi d x=\int_{\Omega} f\left(x, u,|\nabla w|^{p(x)-2} \nabla w\right) \varphi d x
$$

where $\varphi \in W_{0}^{1, p(x)}(\Omega)$.

Consider the following functional

$$
J(u)=\int_{\Omega} \frac{|\nabla u|^{p(x)}+|u|^{p(x)}}{p(x)} d x, \forall u \in W_{0}^{1, p(x)}(\Omega) .
$$

and $L=J^{\prime}: W_{0}^{1, p(x)}(\Omega) \rightarrow\left(W_{0}^{1, p(x)}(\Omega)\right)^{*}$, namely,

$$
(L(u), v)=\int_{\Omega}\left(|\nabla u|^{p(x)-2} \nabla u \nabla v+|u|^{p(x)-2} u v\right) d x, \forall u, v \in W_{0}^{1, p(x)}(\Omega) .
$$

Our proof is based on the famous Mountain Pass Lemma.

Lemma A.[23] Let $E$ be a real Banach space, and $I \in C^{1}(E, \mathbb{R})$ satisfies $(P S)$ condition. Suppose

(i) there exists constants $\rho>0, \alpha>0$ such that

$$
\left.I\right|_{\partial B \rho} \geq I(0)+\alpha
$$

with $B_{\rho}=\left\{u \in W_{0}^{1, p(x)}(\Omega):\|u\| \leq \rho\right\}$;

(ii) there is an $e \in E$ and $\|e\|>\rho$ such that $I(e) \leq I(0)$. 
R. Ayazoglu (Mashiyev), S. Akbulutb, E. Akkoyunluc - Solutions for ...

Then $I(u)$ has a critical value $c$ which can be characterized as

$$
c_{w}=\inf _{\gamma \in \Gamma} \max _{u \in \gamma([0,1])} I(u)
$$

, where

$$
\Gamma=\{\gamma \in C([0,1], E): \gamma(0)=0, \gamma(1)=e\} .
$$

Remark 3.1 A functional $I$ satisfies the Palais-Smale $(P S)$ condition for short, we mean that if any sequence $\left\{u_{n}\right\}$ in $E$ such that $\left\{I\left(u_{n}\right)\right\}$ bounded and $I^{\prime}\left(u_{n}\right) \rightarrow 0$ as $n \rightarrow \infty$,admits a convergent subsequence.

Lemma 3.1. Let $w \in W_{0}^{1, p(x)}(\Omega)$. Then

(1) there exists constants $\rho>0, \alpha>0$ such that $\left.I_{w}\right|_{\partial B \rho} \geq \alpha$ with $B_{\rho}=\left\{u \in W_{0}^{1, p(x)}(\Omega):\|u\| \leq \rho\right\}$

(2) for $\sigma \in C_{0}^{\infty}(\Omega)$ with $\|\sigma\|=1, I_{w}(t \sigma) \rightarrow-\infty$ as $t \rightarrow \infty$.

Proof. (1). Let $\|u\|<1$. From $\left(\mathbf{f}_{2}\right)$ and $\left(\mathbf{f}_{3}\right)$, there exists a positive constant $C_{1}$, independent of $w$, such that

$$
F\left(x, t,|\xi|^{p(x)-2} \xi\right) \leq \frac{1}{2 p^{+}}|t|^{p(x)}+C_{1}|t|^{q(x)} .
$$

Then by Proposition 2.2 and Proposition 2.5, we have

$$
\begin{aligned}
I_{w}(u) & =\int_{\Omega} \frac{|\nabla u|^{p(x)}+|u|^{p(x)}}{p(x)} d x-\int_{\Omega} F\left(x, u,|\nabla w|^{p(x)-2} \nabla w\right) d x \\
& \geq \frac{1}{p^{+}} \int_{\Omega}\left(|\nabla u|^{p(x)}+|u|^{p(x)}\right) d x-\frac{1}{2 p^{+}} \int_{\Omega}|u|^{p(x)} d x-C_{1} \int_{\Omega}|u|^{q(x)} d x \\
& \geq \frac{1}{2 p^{+}}\|u\|^{p^{+}}-C_{1} \max \left\{|u|^{q^{-}},|u|^{q^{+}}\right\} \\
& \geq \frac{1}{2 p^{+}}\|u\|^{p^{+}}-C_{2}\left(\|u\|^{q^{-}}+\|u\|^{q^{+}}\right) .
\end{aligned}
$$

Since $p^{+}<q^{-}$, there exist two positive real numbers $\rho$ and $\alpha$ such that $I_{w}(u) \geq \alpha>0$, $u \in W_{0}^{1, p(x)}(\Omega)$ with $\|u\| \leq \rho$. First part of Lemma 3.1 holds.

$(2)$. Taking an arbitrary $v_{0} \in W_{0}^{1, p(x)}(\Omega) /\{0\}$ and from $\left(\mathbf{f}_{5}\right)$, we have

$$
\begin{aligned}
I_{w}\left(t v_{0}\right) & \leq \int_{\Omega} \frac{\left|\nabla t v_{0}\right|^{p(x)}+\left|t v_{0}\right|^{p(x)}}{p(x)} d x-\int_{\Omega} F\left(x, t v_{0},|\nabla w|^{p(x)-2} \nabla w\right) d x \\
& \leq \frac{t^{p^{+}}}{p^{-}} \int_{\Omega}\left(\left|\nabla v_{0}\right|^{p(x)}+\left|v_{0}\right|^{p(x)}\right) d x-a_{1} t^{\theta} \int_{\Omega}\left|v_{0}\right|^{\theta} d x+a_{2}|\Omega| .
\end{aligned}
$$

Since $\theta>p^{+}$and $\left|v_{0}\right|^{\theta} \neq 0$ then $I_{w}\left(t v_{0}\right) \rightarrow-\infty$ as $t \rightarrow \infty$. 
R. Ayazoglu (Mashiyev), S. Akbulutb, E. Akkoyunluc - Solutions for ...

Therefore, the second part of Lemma 3.1 is proved.

The Mountain Pass theorem (see, e.g. [19,24]) implies the existence of a sequence $\left\{u_{n}\right\} \subset W_{0}^{1, p(x)}(\Omega)$ such that

$$
I_{w}\left(u_{n}\right) \rightarrow c_{w} \text { and } I_{w}^{\prime}\left(u_{n}\right) \rightarrow 0 .
$$

Lemma 3.2. Let $w \in W_{0}^{1, p(x)}(\Omega)$. Then functional $I_{w}$ satisfies Palais-Smale $(P S)$ condition.

Proof. First, we show that $\left\{u_{n}\right\}$ is bounded in $W_{0}^{1, p(x)}(\Omega)$. Assume by contradiction the contrary. Then, passing eventually to a subsequence, still denoted by $\left\{u_{n}\right\}$, we may assume that $\left\|u_{n}\right\| \rightarrow 0$ as $n \rightarrow \infty$. By $\left(\mathbf{f}_{4}\right)$ and (3.4) imply that for $n$ large enough it holds

$$
\begin{aligned}
& 1+c_{w}+\left\|u_{n}\right\| \\
\geq & I_{w}\left(u_{n}\right)-\frac{1}{\theta}\left\langle I_{w}^{\prime}\left(u_{n}\right), u_{n}\right\rangle \\
\geq & \frac{1}{p^{+}} \int_{\Omega}\left(\left|\nabla u_{n}\right|^{p(x)}+\left|u_{n}\right|^{p(x)}\right) d x-\int_{\Omega} F\left(x, u_{n},|\nabla w|^{p(x)-2} \nabla w\right) d x \\
& -\frac{1}{\theta} \int_{\Omega}\left(\left|\nabla u_{n}\right|^{p(x)}+\left|u_{n}\right|^{p(x)}\right) d x-\int_{\Omega} \frac{1}{\theta} u_{n} f\left(x, u_{n},|\nabla w|^{p(x)-2} \nabla w\right) d x \\
\geq & \left(\frac{1}{p^{+}}-\frac{1}{\theta}\right) \int_{\Omega}\left(\left|\nabla u_{n}\right|^{p(x)}+\left|u_{n}\right|^{p(x)}\right) d x \\
& -\int_{\left\{x \in \Omega: u_{n}(x) \geq t_{0}\right\}}\left[\frac{1}{\theta} u_{n} f\left(x, u_{n},|\nabla w|^{p(x)-2} \nabla w\right)-F\left(x, u_{n},|\nabla w|^{p(x)-2} \nabla w\right)\right] d x \\
& -\int_{\left\{x \in \Omega: u_{n}(x)<t_{0}\right\}}\left[\frac{1}{\theta} u_{n} f\left(x, u_{n},|\nabla w|^{p(x)-2} \nabla w\right)-F\left(x, u_{n},|\nabla w|^{p(x)-2} \nabla w\right)\right] d x \\
\geq & \left(\frac{1}{p^{+}}-\frac{1}{\theta}\right)\left\|u_{n}\right\|^{p^{-}}-\int_{\left\{x \in \Omega: u_{n}(x) \geq t_{0}\right\}}\left[\frac{1}{\theta} u_{n} f\left(x, u_{n},|\nabla w|^{p(x)-2} \nabla w\right)-F\left(x, u_{n},|\nabla w|^{p(x)-2} \nabla w\right)\right] d x \\
& -M|\Omega|,
\end{aligned}
$$

where $M=\sup \left\{\frac{1}{\theta} t f\left(x, t,|\nabla w|^{p(x)-2} \nabla w\right)-F\left(x, t,|\nabla w|^{p(x)-2} \nabla w\right),|t|<t_{0}\right\}$. Taking into account that condition $\left(\mathbf{f}_{4}\right)$ holds true, dividing the above inequality by $\left\|u_{n}\right\|$ and passing to the limit as $n \rightarrow \infty$ we obtain a contradiction. It follows that $\left\{u_{n}\right\}$ is bounded in $W_{0}^{1, p(x)}(\Omega)$.

Let $g(u)=\int_{\Omega} F(x, u, \cdot) d x$, then $g^{\prime}\left(u_{n}\right) \rightarrow g^{\prime}(u)$. Since $I_{w}^{\prime}\left(u_{n}\right)=L\left(u_{n}\right)-g^{\prime}\left(u_{n}\right) \rightarrow 0$, we have $L\left(u_{n}\right) \rightarrow g^{\prime}\left(u_{n}\right)$. From Proposition 2.6 it follows that $u_{n} \rightarrow u$. Therefore, $I_{w}$ satisfies Palais-Smale $(P S)$ condition.

Lemma 3.3 Assume the conditions $\left(\mathbf{f}_{1}\right)-\left(\mathbf{f}_{7}\right)$ hold. If in addition $p$ also satisfies (3.3), then problem $\left(\mathbf{P}_{w}\right)$ has at least one positive solution $u_{w} \in C^{1, \alpha}(\Omega)$ with $\alpha \in(0,1)$, for any 
$w \in W_{0}^{1, p(x)}(\Omega) \cap C^{1, \alpha}(\Omega)$. Further, there exist positive constants $\rho_{1}$ and $\rho_{2}$, independent of $w$, such that $\left|u_{w}\right|_{C^{0, \alpha}(\Omega)} \leq \rho_{1}$ and $\left|\nabla u_{w}\right|_{C^{0, \alpha}(\Omega)} \leq \rho_{2}$.

Proof. Lemma 3.1 and Lemma 3.2 imply that the functional $I_{w}$ satisfies the MountainPass theorem implies the existence of a sequence $\left\{u_{n}\right\} \subset W_{0}^{1, p(x)}(\Omega)$ such that

$$
I_{w}\left(u_{n}\right) \rightarrow c_{w} \text { and } I_{w}^{\prime}\left(u_{n}\right) \rightarrow 0
$$

where

$$
c_{w}=\inf _{\gamma \in \Gamma} \max _{t \in[0,1]} I_{w}(\gamma(t))>0
$$

and

$$
\Gamma=\left\{\gamma \in C\left([0,1], W_{0}^{1, p(x)}(\Omega)\right): \gamma(0)=0, \gamma(1)=T v_{0}\right\},
$$

for some $v_{0}$ and $T$ given in Lemma 3.2. Since $\left\{u_{n}\right\}$ is bounded in $W_{0}^{1, p(x)}(\Omega)$ and from Proposition $2.5(i)$ we deduce that there exists a subsequence, again denoted by $\left\{u_{n}\right\}$, and

$$
\begin{array}{cc}
u_{n} \rightarrow u_{w} \text { (weakly) } & \text { in } W_{0}^{1, p(x)}(\Omega), \\
u_{n} \rightarrow u_{w} \text { (strongly) } & \text { in } L^{p(x)}(\Omega) \text { for } p(x)<p^{*}(x), \\
u_{n}(x) \rightarrow u_{w}(x) & \text { a.e. in } \Omega,
\end{array}
$$

and also, arguing as $[12,13,14]$, we have $\frac{\partial u_{n}}{\partial x_{i}}(x) \rightarrow \frac{\partial u_{w}}{\partial x_{i}}(x)$ a.e. in $\Omega$. Further, using the results argued in [4], we have

$$
\int_{\Omega}\left|\nabla u_{n}\right|^{p(x)-2} \nabla u_{n} \nabla \varphi d x \rightarrow \int_{\Omega}\left|\nabla u_{w}\right|^{p(x)-2} \nabla u_{w} \nabla \varphi d x
$$

and

$$
\int_{\Omega}\left|u_{n}\right|^{p(x)-2} u_{n} \varphi d x \rightarrow \int_{\Omega}\left|u_{w}\right|^{p(x)-2} u_{w} \varphi d x
$$

for all $\varphi \in W_{0}^{1, p(x)}(\Omega)$. Moreover, using again [4] and Lebesgue generalized theorem [5], we get

$$
\int_{\Omega} f\left(x, u_{n},|\nabla w|^{p(x)-2} \nabla w\right) \varphi d x \rightarrow \int_{\Omega} f\left(x, u_{w},|\nabla w|^{p(x)-2} \nabla w\right) \varphi d x,
$$

for all $\varphi \in W_{0}^{1, p(x)}(\Omega)$. Hence, we obtain that $\left\langle I_{w}^{\prime}\left(u_{w}\right), \varphi\right\rangle=0$ for all $\varphi \in W_{0}^{1, p(x)}(\Omega)$. From $\left(\mathbf{f}_{2}\right)$ and $\left(\mathbf{f}_{3}\right)$, given $\varepsilon>0$ there exists a positive constant $C_{\varepsilon}>0$, independent of $w$, such that $\left|f\left(x, t,|\xi|^{p(x)-2} \xi\right)\right| \leq \varepsilon|t|^{p(x)-1}+C_{\varepsilon}|t|^{q(x)-1}$ for all $\xi \in \mathbb{R}^{N}$. On the other hand, since $q \in C_{+}(\bar{\Omega})$ such that $q(x)<p^{*}(x) \forall x \in \bar{\Omega}$, by Theorem A $(a)$ we have $u_{w} \in L^{\infty}(\Omega)$, and hence $u_{w} \in W^{1, p(x)}(\Omega) \cap L^{\infty}(\Omega)$. Moreover, since the function $p$ is Hölder continuous on $\bar{\Omega}$ then by Theorem A $(c)$ we get $u_{w} \in C^{1, \alpha}(\Omega)$ with $\alpha \in(0,1)$ $\forall w \in W_{0}^{1, p(x)}(\Omega) \cap C^{1, \alpha}(\Omega)$. 
R. Ayazoglu (Mashiyev), S. Akbulutb, E. Akkoyunluc - Solutions for ...

Remark 3.2. We want to note that, if the assumption (3.3) is replaced by (3.2) in Lemma 3.3 , then by Theorem A $(b)$ one concludes that $u_{w} \in C^{0, \alpha}(\Omega)$ with $\alpha \in(0,1)$, $\forall w \in W_{0}^{1, p(x)}(\Omega) \cap C^{1, \alpha}(\Omega)$.

Lemma 3.4. Let $w \in W_{0}^{1, p(x)}(\Omega)$. There exists a positive constant $C_{*}$, independent of $w$, such that $\left\|u_{w}\right\| \geq C_{*}$, for all solutions $u_{w}$ obtained in Lemma 3.3.

Proof. Since $u_{w} \neq 0$ is a solution of problem $\left(\mathbf{P}_{w}\right)$, we have

$$
\int_{\Omega} \frac{\left|\nabla u_{w}\right|^{p(x)}+\left|u_{w}\right|^{p(x)}}{p(x)} d x=\int_{\Omega} f\left(x, u_{w},|\nabla w|^{p(x)-2} \nabla w\right) u_{w} d x .
$$

Then, from $\left(\mathbf{f}_{2}\right)$ and $\left(\mathbf{f}_{3}\right)$, there exists a positive constant $C_{3}$, independent of $w$, such that $\left|f\left(x, t,|\xi|^{p(x)-2} \xi\right)\right| \leq \frac{1}{2 p^{+}}|t|^{p(x)-1}+C_{3}|t|^{q(x)-1}$ for all $\xi \in \mathbb{R}^{N}$. It is sufficient to consider only the case $\left\|u_{w}\right\|<1$. Thus, by Proposition 2.2 and Proposition 2.5, we have

$$
\begin{aligned}
\frac{1}{2 p^{+}} \int_{\Omega}\left|u_{w}\right|^{p(x)} d x+C_{3} \int_{\Omega}\left|u_{w}\right|^{q(x)} d x & \geq \frac{1}{p^{+}} \int_{\Omega}\left(|\nabla u|^{p(x)}+|u|^{p(x)}\right) d x \\
C_{3} \int_{\Omega}\left|u_{w}\right|^{q(x)} d x & \geq \frac{1}{2 p^{+}}\left\|u_{w}\right\|^{p^{+}} \\
C_{4}\left\|u_{w}\right\|^{q^{-}} & \geq \frac{1}{2 p^{+}}\left\|u_{w}\right\|^{p^{+}} \\
\left\|u_{w}\right\| & \geq\left(\frac{1}{2 p^{+} C_{4}}\right)^{1 /\left(q^{-}-p^{+}\right)}:=C_{*} .
\end{aligned}
$$

The proof is complete.

Lemma 3.5. Let $w \in W_{0}^{1, p(x)}(\Omega)$. There exists a positive constant $C^{*}$, independent of $w$, such that $\left\|u_{w}\right\| \leq C^{*}$, for all solutions $u_{w}$ obtained in Lemma 3.4.

Proof. Notice that

$$
I_{w}\left(u_{w}\right) \leq \max _{t \geq 0} I_{w}\left(t v_{0}\right)
$$

with $v_{0}$ given as in Lemma 3.2. we get

$$
\begin{aligned}
& I_{w}\left(u_{w}\right) \\
\leq & \frac{t^{p^{+}}}{p^{-}} \int_{\Omega}\left(\left|\nabla v_{0}\right|^{p(x)}+\left|v_{0}\right|^{p(x)}\right) d x-a_{1} t^{\theta} \int_{\Omega}\left|v_{0}\right|^{\theta} d x-a_{2}|\Omega| .
\end{aligned}
$$

Since $\theta>p^{+}$and $\left|v_{0}\right|^{\theta} \neq 0$, the map

$$
t \in \mathbb{R} \longmapsto \frac{t^{p^{+}}}{p^{-}} \int_{\Omega}\left(\left|\nabla v_{0}\right|^{p(x)}+\left|v_{0}\right|^{p(x)}\right) d x-a_{1} t^{\theta} \int_{\Omega}\left|v_{0}\right|^{\theta} d x-a_{2}|\Omega|
$$


attains a positive maximum, independent of $w$. So we get a constant $C>0$ such that

$$
I_{w}\left(u_{w}\right) \leq C
$$

By (3.6), we have

$$
\int_{\Omega} \frac{\left|\nabla u_{w}\right|^{p(x)}+\left|u_{w}\right|^{p(x)}}{p(x)} d x \leq C+\int_{\Omega} F\left(x, u_{w},|\nabla w|^{p(x)-2} \nabla w\right) d x .
$$

Define $G=\left\{x \in \Omega:\left|u_{w}\right|>t_{0}>1\right\}$, where $t_{0}$ is given in $\left(\mathbf{f}_{4}\right)$. Keeping in mind that $u_{w}$ is a solution from $\left(\mathbf{f}_{2}\right)$ and $\left(\mathbf{f}_{4}\right)$, we have

$$
\begin{aligned}
& \int_{\Omega} F\left(x, u_{w},|\nabla w|^{p(x)-2} \nabla w\right) d x \\
\leq & \int_{\Omega \backslash G} F\left(x, u_{w},|\nabla w|^{p(x)-2} \nabla w\right) d x+\int_{G} F\left(x, u_{w},|\nabla w|^{p(x)-2} \nabla w\right) d x \\
\leq & C_{5}\left(t_{0}+\frac{\left|t_{0}\right|^{p(x)}}{p(x)}\right)|\Omega \backslash G|+\int_{\Omega} \frac{\left|\nabla u_{w}\right|^{p(x)}}{\theta} d x \\
\leq & C_{5}\left(t_{0}+\frac{\left|t_{0}\right|^{p^{+}}}{p^{-}}\right)|\Omega \backslash G|+\int_{\Omega} \frac{\left|\nabla u_{w}\right|^{p(x)}+\left|u_{w}\right|^{p(x)}}{\theta} d x .
\end{aligned}
$$

Returning to equation (3.7), we have

$$
\begin{aligned}
& \left(\frac{1}{p^{+}}-\frac{1}{\theta}\right) \int_{\Omega}\left(\left|\nabla u_{w}\right|^{p(x)}+\left|u_{w}\right|^{p(x)}\right) d x \\
\leq & C+C_{5}\left(t_{0}+\frac{\left|t_{0}\right|^{p^{+}}}{p^{-}}\right)|\Omega \backslash G|,
\end{aligned}
$$

where $|\Omega \backslash G|$ denotes the Lebesgue measure in $\mathbb{R}^{N}$ of the set $\Omega \backslash G$. Furthermore, we obtain

$$
\left(\frac{1}{p^{+}}-\frac{1}{\theta}\right)\left\|u_{w}\right\|^{p^{-}} \leq C+C_{5}\left(t_{0}+\frac{\left|t_{0}\right|^{p^{+}}}{p^{-}}\right)|\Omega \backslash G|:=\widetilde{C}^{*} .
$$

Thus,

$$
\left\|u_{w}\right\| \leq\left[\left(\frac{1}{p^{+}}-\frac{1}{\theta}\right)^{-1} \widetilde{C}^{*}\right]^{1 / p^{-}}:=C^{*} .
$$

The proof is complete.

Now we are ready to show that problem $(\mathbf{P})$ has a positive solution. 
R. Ayazoglu (Mashiyev), S. Akbulutb, E. Akkoyunluc - Solutions for ... of

Proof of Theorem 3.1. We consider a sequence $\left\{u_{n}\right\} \subset W_{0}^{1, p(x)}(\Omega) \cap C^{1, \alpha}(\Omega)$ as solutions

$$
\left\{\begin{array}{c}
-\Delta_{p(x)} u_{n}+\left|u_{n}\right|^{p(x)-2} u_{n}=f\left(x, u_{n},\left|\nabla u_{n-1}\right|^{p(x)-2} \nabla u_{n-1}\right) \text { in } \Omega \\
u_{n}=0 \text { on } \partial \Omega
\end{array}\right.
$$

obtained by the Mountain Pass Theorem in Lemma 3.3, starting with an arbitrary $u_{0} \in$ $W_{0}^{1, p(x)}(\Omega) \cap C^{1, \alpha}(\Omega),\left|u_{w}\right|_{C^{0}(\Omega)} \leq \rho_{1}$ and $\left|\nabla u_{w}\right|_{C^{0}(\Omega)} \leq \rho_{2}$. On the other hand, using $(\mathbf{P})_{n+1}$ and $(\mathbf{P})_{n}$, we obtain the followings

$$
\begin{aligned}
& \int_{\Omega}\left|\nabla u_{n+1}\right|^{p(x)-2} \nabla u_{n+1}\left(\nabla u_{n+1}-\nabla u_{n}\right) d x+\int_{\Omega}\left|u_{n+1}\right|^{p(x)-2} u_{n+1}\left(u_{n+1}-u_{n}\right) d x \\
= & \int_{\Omega} f\left(x, u_{n+1},\left|\nabla u_{n}\right|^{p(x)-2} \nabla u_{n}\right)\left(u_{n+1}-u_{n}\right) d x
\end{aligned}
$$

and

$$
\begin{aligned}
& \int_{\Omega}\left|\nabla u_{n}\right|^{p(x)-2} \nabla u_{n}\left(\nabla u_{n+1}-\nabla u_{n}\right) d x+\int_{\Omega}\left|u_{n}\right|^{p(x)-2} u_{n}\left(u_{n+1}-u_{n}\right) d x \\
= & \int_{\Omega} f\left(x, u_{n},\left|\nabla u_{n-1}\right|^{p(x)-2} \nabla u_{n-1}\right)\left(u_{n+1}-u_{n}\right) d x .
\end{aligned}
$$

Then

$$
\begin{aligned}
& \int_{\Omega}\left(\left|\nabla u_{n+1}\right|^{p(x)-2} \nabla u_{n+1}-\left|\nabla u_{n}\right|^{p(x)-2} \nabla u_{n}\right)\left(\nabla u_{n+1}-\nabla u_{n}\right) d x \\
& +\int_{\Omega}\left(\left|u_{n+1}\right|^{p(x)-2} u_{n+1}-\left|u_{n}\right|^{p(x)-2} u_{n}\right)\left(u_{n+1}-u_{n}\right) d x \\
= & \int_{\Omega}\left(f\left(x, u_{n+1},\left|\nabla u_{n}\right|^{p(x)-2} \nabla u_{n}\right)-f\left(x, u_{n},\left|\nabla u_{n}\right|^{p(x)-2} \nabla u_{n}\right)\right)\left(u_{n+1}-u_{n}\right) d x \\
& +\int_{\Omega}\left(f\left(x, u_{n},\left|\nabla u_{n}\right|^{p(x)-2} \nabla u_{n}\right)-f\left(x, u_{n},\left|\nabla u_{n-1}\right|^{p(x)-2} \nabla u_{n-1}\right)\right)\left(u_{n+1}-u_{n}\right) d x .
\end{aligned}
$$


From Proposition 2.2 and Proposition 2.4, we get

$$
\begin{aligned}
& \int_{\Omega}|\eta-\psi|^{p(x)} d x \\
\leq & 2\left\|\frac{|\eta-\psi|^{p(x)}}{(|\eta|+|\psi|)^{\frac{p(x)(2-p(x))}{2}}}\right\|_{L^{\frac{2}{p(x)}}(\Omega)}\left\|(|\eta|+|\psi|)^{\frac{p(x)(2-p(x))}{2}}\right\|_{L^{\frac{2}{2-p(x)}(\Omega)}} \\
\leq & 2 \max _{p \in\left\{p^{-}, p^{+}\right\}}\left\|\frac{|\eta-\psi|^{2}}{(|\eta|+|\psi|)^{2-p(x)}}\right\|_{L^{\frac{2}{p(x)}} \cdot \frac{p(x)}{2}}^{(\Omega)}\left\|(|\eta|+|\psi|)^{p(x)}\right\|_{L^{\frac{2}{2-p(x)}} \cdot \frac{2-p(x)}{2}}^{(2-p) / 2}(\Omega) \\
= & 2 \max _{p \in\left\{p^{-}, p^{+}\right\}}\left(\int_{\Omega} \frac{|\eta-\psi|^{2}}{(|\eta|+|\psi|)^{2-p(x)}} d x\right)^{p / 2}\left(\int_{\Omega}(|\eta|+|\psi|)^{p(x)} d x\right)^{(2-p) / 2} \\
\leq & 2 \max _{p \in\left\{p^{-}, p^{+}\right\}}\left(\int_{\Omega} \frac{|\eta-\psi|^{2}}{(|\eta|+|\psi|)^{2-p(x)}} d x\right)^{p / 2}\left(1+\int_{\Omega}(|\eta|+|\psi|)^{p(x)} d x\right)^{1 / 2}
\end{aligned}
$$

Similarly

$$
\begin{aligned}
& \int_{\Omega}|\nabla \eta-\nabla \psi|^{p(x)} d x \\
\leq & 2 \max _{p \in\left\{p^{-}, p^{+}\right\}}\left(\int_{\Omega} \frac{|\nabla \eta-\nabla \psi|^{2}}{(|\nabla \eta|+|\nabla \psi|)^{2-p(x)}} d x\right)^{p / 2}\left(1+\int_{\Omega}\left(|\nabla \eta|^{p(x)}+|\nabla \psi|^{p(x)}\right) d x\right)^{1 / 2} .
\end{aligned}
$$

Considering the assumption $1<p^{-} \leq p^{+}<2$, and applying (3.1) we obtain

$$
\begin{aligned}
& \max _{p \in\left\{p^{-}, p^{+}\right\}}\left(\int_{\Omega} \frac{|\eta-\psi|^{2}}{(|\eta|+|\psi|)^{2-p(x)}} d x\right)^{p / 2} \\
\leq & \max _{p \in\left\{p^{-}, p^{+}\right\}}\left(\frac{1}{p^{-}-1} \int_{\Omega}\left(|\eta|^{p(x)-2} \eta-|\psi|^{p(x)-2} \psi\right) \cdot(\eta-\psi) d x\right)^{p / 2} \\
\leq & \frac{1}{p^{-}-1}\left(1+\int_{\Omega}\left(|\eta|^{p(x)-2} \eta-|\psi|^{p(x)-2} \psi\right) \cdot(\eta-\psi) d x\right),
\end{aligned}
$$

and

$$
\begin{aligned}
& \max _{p \in\left\{p^{-}, p^{+}\right\}} \int_{\Omega} \frac{|\nabla \eta-\nabla \psi|^{2}}{(|\nabla \eta|+|\nabla \psi|)^{2-p(x)}} d x \\
\leq & \frac{1}{p^{-}-1}\left(1+\int_{\Omega}\left(|\nabla \eta|^{p(x)-2} \nabla \eta-|\nabla \psi|^{p(x)-2} \nabla \psi\right) \cdot(\nabla \eta-\nabla \psi) d x\right) .
\end{aligned}
$$


Let $1+\left(\int_{\Omega}(|\eta|+|\psi|)^{p(x)} d x\right)^{\frac{1}{2}}:=L_{3}$ and $1+\left(\int_{\Omega}(|\nabla \eta|+|\nabla \psi|)^{p(x)} d x\right)^{\frac{1}{2}}:=L_{4}$. Then $L_{3}, L_{4}$ is bounded and $L_{3}, L_{4} \geq 1$. Therefore,

$$
\begin{aligned}
& \int_{\Omega}|\eta-\psi|^{p(x)} d x \\
\leq & \frac{2 L_{3}}{p^{-}-1}+\frac{2 L_{3}}{p^{-}-1} \int_{\Omega}\left(|\eta|^{p(x)-2} \eta-|\psi|^{p(x)-2} \psi\right) \cdot(\eta-\psi) d x,
\end{aligned}
$$

and

$$
\begin{aligned}
& \int_{\Omega}|\nabla \eta-\nabla \psi|^{p(x)} d x \\
\leq & \frac{2 L_{4}}{p^{-}-1}+\frac{2 L_{4}}{p^{-}-1} \int_{\Omega}\left(|\nabla \eta|^{p(x)-2} \nabla \eta-|\nabla \psi|^{p(x)-2} \nabla \psi\right) \cdot(\nabla \eta-\nabla \psi) d x .
\end{aligned}
$$

Thus, if we choose $M|\Omega|=\frac{2\left(L_{3}+L_{4}\right)}{p^{-}-1}$, we have

$$
\begin{aligned}
& \widetilde{\rho}_{p(x)}\left(u_{n+1}-u_{n}\right)=\int_{\Omega}\left(\left|u_{n+1}-u_{n}\right|^{p(x)}+\left|\nabla u_{n+1}-\nabla u_{n}\right|^{p(x)}\right) d x \\
\leq & \frac{2\left(L_{3}+L_{4}\right)}{\left(p^{-}-1\right)}+\frac{2 L_{1} L_{3}}{p^{-}-1} \rho_{p(x)}\left(u_{n+1}-u_{n}\right) \\
& +\frac{2 L_{2} L_{4}}{p^{-}-1} \int_{\Omega}\left|\nabla u_{n}-\nabla u_{n-1}\right|^{p(x)-1}\left|u_{n+1}-u_{n}\right| d x-M|\Omega| \\
= & \frac{2 L_{1} L_{3}}{p^{-}-1} \rho_{p(x)}\left(u_{n+1}-u_{n}\right)+\frac{2 L_{2} L_{4}}{p^{-}-1} \int_{\Omega}\left|\nabla u_{n}-\nabla u_{n-1}\right|^{p(x)-1}\left|u_{n+1}-u_{n}\right| d x .
\end{aligned}
$$

Applying Proposition 2.3 to the right-hand side of the above inequality, we get

$$
\begin{aligned}
& \widetilde{\rho}_{p(x)}\left(u_{n+1}-u_{n}\right) \\
\leq & \frac{2 L_{1} L_{3}}{p^{-}-1} \rho_{p(x)}\left(u_{n+1}-u_{n}\right) \\
& +\frac{2 L_{2} L_{4}}{p^{-}-1}\left[\frac{1}{p^{-}} \rho_{p(x)}\left(u_{n+1}-u_{n}\right)+\frac{p^{+}-1}{p^{-}} \rho_{p(x)}\left(u_{n}-u_{n-1}\right)\right] \\
\leq & \left(\frac{2 L_{1} L_{3}}{p^{-}-1}+\frac{2 L_{2} L_{4}}{\left(p^{-}-1\right) p^{-}}\right) \rho_{p(x)}\left(u_{n+1}-u_{n}\right) \\
& +\frac{2 L_{2} L_{4}\left(p^{+}-1\right)}{p^{-}\left(p^{-}-1\right)} \rho_{p(x)}\left(u_{n}-u_{n-1}\right) \\
\leq & \frac{2 L_{1} L_{3} p^{-}+2 L_{2} L_{4}}{\left(p^{-}-1\right) p^{-}} \widetilde{\rho}_{p(x)}\left(u_{n+1}-u_{n}\right)+\frac{2 L_{2} L_{4}\left(p^{+}-1\right)}{p^{-}\left(p^{-}-1\right)} \widetilde{\rho}_{p(x)}\left(u_{n}-u_{n-1}\right)
\end{aligned}
$$

or

$$
1-\frac{2 L_{1} L_{3} p^{-}+2 L_{2} L_{4}}{p^{-}\left(p^{-}-1\right)} \widetilde{\rho}_{p(x)}\left(u_{n+1}-u_{n}\right) \leq \frac{2 L_{2} L_{4}\left(p^{+}-1\right)}{p^{-}\left(p^{-}-1\right)} \widetilde{\rho}_{p(x)}\left(u_{n}-u_{n-1}\right) .
$$


From (3.8), $1-\frac{2 L_{1} L_{3} p^{-}+2 L_{2} L_{4}}{\left(p^{-}-1\right) p^{-}}>0$ holds. Thus

$$
\widetilde{\rho}_{p(x)}\left(u_{n+1}-u_{n}\right) \leq \frac{2 L_{2} L_{4}\left(p^{+}-1\right)}{p^{-}\left(p^{-}-1\right)-2\left(L_{1} L_{3} p^{-}+L_{2} L_{4}\right)} \widetilde{\rho}_{p(x)}\left(u_{n}-u_{n-1}\right) .
$$

Let $\frac{2 L_{2} L_{4}\left(p^{+}-1\right)}{p^{-}\left(p^{-}-1\right)-\left(2 L_{1} L_{3} p^{-}+2 L_{2} L_{4}\right)}:=K$. According to (3.4), we have $K<1$. Now, applying the triangle inequality consecutively, we get

$$
\begin{aligned}
& \int_{\Omega}\left|\nabla u_{n+k}-\nabla u_{n}\right|^{p(x)} d x \\
\leq & \left(2^{p^{+}-1} K^{n+k-1}+2^{2\left(p^{+}-1\right)} K^{n+k-2}+\cdots+2^{(k-1)\left(p^{+}-1\right)} K^{n}\right) \int_{\Omega}\left|\nabla u_{1}-\nabla u_{0}\right|^{p(x)} d x \\
\leq & \left(2^{(k-1)\left(p^{+}-1\right)} K^{n+k-1}+2^{(k-1)\left(p^{+}-1\right)} K^{n+k-2}+\cdots+2^{(k-1)\left(p^{+}-1\right)} K^{n}\right) \int_{\Omega}\left|\nabla u_{1}-\nabla u_{0}\right|^{p(x)} d x \\
\leq & 2^{(k-1)\left(p^{+}-1\right)} \frac{1-K^{k}}{1-K} K^{n} \int_{\Omega}\left|\nabla u_{1}-\nabla u_{0}\right|^{p(x)} d x .
\end{aligned}
$$

Therefore, we obtain that

$$
\widetilde{\rho}_{p(x)}\left(u_{n+k}-u_{n}\right) \leq 2^{(k-1)\left(p^{+}-1\right)+1} \frac{1-K^{k}}{1-K} K^{n} \widetilde{\rho}_{p(x)}\left(u_{1}-u_{0}\right) .
$$

Since $\lim _{n \rightarrow \infty} K^{n}=0$, using Proposition 2.1 we have

$$
\lim _{n \rightarrow \infty}\left\|u_{n+k}-u_{n}\right\|=0
$$

Therefore, it follows that the sequence $\left\{u_{n}\right\}$ strongly converges in $W_{0}^{1, p(x)}(\Omega)$ to some function $u \in W_{0}^{1, p(x)}(\Omega)$, as it easily follows proving that $\left\{u_{n}\right\}$ is a Cauchy sequence in $W_{0}^{1, p(x)}(\Omega)$. Since $\left\|u_{n}\right\| \geq C_{*}$ for all $n$, we have that $u>0$ in $W_{0}^{1, p(x)}(\Omega)$.

\section{REFERENCES}

[1] A. Ambrosetti and P. H. Rabinowitz, Dual variational methods in critical point theory, J. Funct. Anal., 14(1973), 349-381.

[2] R. Ayazoglu(Mashiyev) and Ismail Ekincioglu, Electrorheological Fluids Equations Involving Variable Exponent with Dependence on the Gradient via Mountain Pass Techniques, Numerical Functional Analysis and Optimization, Volume 37, 2016, Issue 9, 1144-1157.

[3] M. M. Boureanu, P. Pucci and V. D. Radulescu, Multiplicity of solutions for a class of anisotropic elliptic equations with variable exponent, Complex Var. Elliptic Equ. 56 (2011), 755-767. 
R. Ayazoglu (Mashiyev), S. Akbulutb, E. Akkoyunluc - Solutions for ...

[4] H. Brezis and E. H. Lieb, A relation between pointwise convergence of functions and convergence functionals, Proc. Am. Math. Soc. 8 (1983), 486-490.

[5] H. Brezis, Analyse Fonctionelle. Théorie et Aplications, Paris, Masson, 1987.

[6] N.T. Chung, Multiple solutions for a class of $p(x)$-Kirchhoff type problems with Neumann boundary conditions, Advances in Pure and Applied Mathematics, 4(2)(2013), 165177.

[7] L. Diening, P. Harjuletho, P. Hästö and M. Růžička, Lebesgue and Sobolev spaces with variable exponents, Springer-Verlag, Berlin, 2011.

[8] X. L. Fan, J. S. Shen and D. Zhao, Sobolev embedding theorems for spaces $W^{k, p(x)}(\Omega)$, J. Math. Anal. Appl. 262 (2001), 749-760.

[9] X. L. Fan and D. Zhao, , On the spaces $L^{p(x)}(\Omega)$ and $W^{m, p(x)}(\Omega)$, J. Math. Anal. Appl., 263(2001), 424-446.

[10] X. L. Fan and D. Zhao, A class of De Giorgi type and Hölder continuity, Nonlinear Anal., 36(1999), 295-318.

[11] X. L. Fan, Global $C^{1, \alpha}$ regularity for variable exponent elliptic equations in divergence form, J. Dif. Equ., 235(2007), 397-417.

[12] D.G. de Figueiredo, M. Girardi and M. Matzeu, Semilinear elliptic equations with dependence on the gradient via Mountain Pass techniques, Diff. Integ. Equ., 17(2004), 119126.

[13] G. M. Figueiredo, Quasilinear equations with dependence on the gradient via Mountain Pass techniques in $\mathbb{R}^{N}$, Applied Mathematics and Comp., 203(2008), 14-18.

[14] J. V. A. Gonçalves and C. O. Alves, Existence of positive solutions for m-Laplacian equations in $\mathbb{R}^{N}$ involving critical Sobolev exponents, Nonlinear Anal., 32(1)(1998), 53-70.

[15] L. Iturriaga and S. Lorca, Existence and multiplicity results of degenerate elliptic equatinos with dependence on the gradient, BVP, Art. ID 47218 (2007), doi:10.1155/2007/47218.

[16] O. Kovăčik and J. Răkosnik, On spaces $L^{p(x)}$ and $W^{k, p(x)}$, Czechoslovak Math., J. 41(116)(1991), 592-618.

[17] D. S. Mitrinović, J. E. Pečaric and A. M. Finj, Classical and New Inequalities in Analysis Kluwer Academic Publishers, Dordrecht, 1993.

[18] M. Montenegro and M. Montenegro, Existence and nonexistence of solutions for quasilinear elliptic equations, J. Math. Anal. Appl., 245(2000), 303-316.

[19] P. Pucci and J. Serrin, Extensions of the mountain pass theorem, J. Funct. Anal., 59(1984), 185-210.

[20] D. Ruiz and A. Suarez, Existence and uniqueness of positive solution of a logistic equation with nonlinear gradient term, Proc. R. Soc. Edinburgh, 137(3)(2007), 555-566.

[21] D. Ruiz, A priori estimates and existence of positive solutions for strongly nonlinear problems, J. Dif. Equ., 199(2004), 96-114. 
R. Ayazoglu (Mashiyev), S. Akbulutb, E. Akkoyunluc - Solutions for ...

[22] M. Růžička, Electrorheological fluids: Modeling and Mathematical Theory, Lecture Notes in Mathematics, Springer-Verlag, Berlin, 2000.

[23] X. Wang and Y. Ding, Existence of multiple solutions to nonlinear elliptic equations in nondivergece form, J. Math. Anal. Appl., 189(1995), 617-630.

[24] M. Willem, Minimax Theorems, Birkhäuser, Basel, 1996.

[25] J.Yin, J. Li, and Y. Ke, Existence of positive solutions for the $p(x)$-Laplacian equation, Rocky Mountain J. Math., (42)(5)(2012), 1421-1758.

R. Ayazoglu (Mashiyev)

Faculty of Educations,

Bayburt University, Turkey

e-mail: rayazoglu@bayburt.edu.tr

Sezgin Akbulutb

Faculty of Science,

Ataturk University, Turkey

e-mail: sezginakbulut@atauni.edu.tr

Ebubekir Akkoyunlu

Bayburt Vocational College,

Bayburt University, Turkey

e-mail: eakkoyunlu@bayburt.edu 